Karczag, Ladislaus, Helgoländer Ufer 7, IV, Berlin

Nolda, Ernst, Ringbahnstr. 21, Halensee

(durch

W. Marckwald und B. Keetman n);

Ramsey, Henrik, Erstes Chemisches Laboratorium der Universität, Berlin (durch O. Aschan und W. Marckwald).

Für die Bibliothek sind als Geschenke eingegangen:

26. v. Fehling, H. Neues Handwörterbuch der Chemie, fortgesetzet von C. Hell und C. Haeussermann. 103. Lieterung. Braunschweig 1907. 106. Jahresbericht über die Fortschritte der Chemie und verwandter Teile anderer Wissenschaften, begründet von J. Liebig und B. Kopp, herausgegeben von W. Kerp und J. Troeger. Für 1901, Heft 4. Braunschweig 1907.

1983. Großmann, H. Die Bedeutung der chemischen Technik für das dentsohe Wirtschaftsleben. Halle a. S. 1907.

1934. Pawlewski, B. Organiczna preparatyka chemiczna. Lemberg 1908. 1935. Kann, A. Die Naturgeschichte der Moral und die Physik des Denkens. Wien 1907.

Der Vorsitzende:

S. Gabriel.
Der Schriftführer:

O. Schotten.

\title{
Auszug aus dem \\ Protokoll der Vorstands-Sitzung
}

vom 4. Dezember 1907.

Anwesend die HHrn. Vorstandsmitglieder: S. Gabriel, A. Bannow, E. Buchner, G. Kraemer, C. Liebermann, F. Mylius, W. Nernst, C. Schotten, H. Wichel ha us, sowie der Generalsekretär Hr. P. Jacobson und der Verwaltungssekretär Hr. R. Stelaner.

53. Hr. E, Buchner berichtet über die am 9 . November d. J. in Berlin stattgehabte Sitzung des $* A$ us schusses zur Wahrung der gemeinsamen Interessen des Chemikerstandes* $\left.{ }^{1}\right)$. Der Vorstand erklärt sich mit der von dem genannten Ausschuß angenommenen Geschäftsordnung einverstanden und delegiert als Vertreter der Deutschen Chemischen Gesellschaft für das Jahr 1908 die HHrn. W. Will

1) vergl. das Protokoll S. 4896-4897 diebes Heftes. 
und $O$. N. Witt; als Ersatzınann für Fälle etwaiger Verhinderung eines Vertreters wird Hr. E. Buchner bestimmt.

Der Vorstand nimmt daron Kenntnis, daß eine Eingabe an das Reichsamt des Innern wegen der Neuregelung der Vorschriften für die Prüfung der Nahrungsmittelcheniker') vom Ausschuß als solchen nicht veraulaßt werden wird, erklärt sich aber bereit, eine solche Eiıgabe in Gemeinschaft mit dem "Verein Deutscher Chemikerk und dem "Verband Selbständiger Öffentlicher Chemiker Deutschlands" zu unterzeichnen.

Der Vorstand nimmt ferner davoll Kenntnis, daß der $>$ Verein Deutscher Chemiker bezüglich der Karenztrage eine Eingabe an den Reichstag und die in Betracht kommenden Behörden eingebracht hat, erklärt indes, daß die Deutsche Cbemische Gesellschaft in Rücksicht auf ihren vorwiegend wissenschaftlichen Charakter nicht in der Lage ist, zu dieser Frage Stellung zu nehmen.

57. Jem Vorstande ist der folgende Antrag, unterzeichnet von einer größeren Anzahl holländischer Mitglieder, zugegangen:

»Die unterzeichneten Mitglieder der Deutschen Chemischen Gesellschait beehren sich, Ihre Aufmerksamkeit auf die hohe Bedeutung ron Formelregistern zu lenken. Sie erlauben sich deshalb Ihnen rorzuschlagen, veranlasseu zu wollen, dab forthin dem Chemischen Zentralblatt ein solches Register beigegeben werde.

Es dürte wohl ïberflüssig sein, nähere Gründe zu diesem Antrage anzuführen, da jeder Uhemiker täglich von dem Chemischen Zentralblatt Gehrauch macht und wohl empfunden haben wird, wie unstïndlich dessen Benutzung ohne Formelregister ist.

Gleichzeitig mōchten sie auch den Wunsch aussprechen, daß den "Berichten* statt am Ende des Jahrgangs, am Schlusse jedes Halbjahres ein Register beigegeben werde. Dadurch würde die bein jetzigen Umfang der $\gg$ Berichte so zeitraubende Arbeit, eine in ersten Halbjahr publizierte Abhandlung in zweiten zurïck zu finden, wesentlich erleichtert werden.«

Der Vorstand sieht sich zurzeit nicht in der Lage, dem ersten Teil des Antrages nähertreten zu können. Denn die Frage der Formelregistrierung ist Ende des Jahres 1901 Gegenstand eingehender Beratungen gewesen '), denen damals ein gemeinschaftlicher Bericht des Zentralblatt-Redakteurs, Hrn. R. A rendt (Leipzig), und des Generalsekretärs, Hrn. P. J acobs on, zugrunde lag. Diese Beratungen haben unter Hinzuziehung des Begründers der Formelregistrierung, Hrn.

1) vergl. diese Berichte 40, 1218, Nr. 5 [1907].

7) vergl. diese Berichte 34, 4389 [1901]. 
M. M. Richter (Karlsrube), dazu geführt, daß die Deutsche Chemische Gesellschaft bis zum Ablauf des Jabres 1911 auf die Ëinführung der Formelregister in das Chemische Zentralblatt verzichtet, vorausgesetzt, daB bis zu diesem Zeitpunkte durch Hrn. Richter die Literatur nach dem Formelsystem unter Hinzufügung des Zentralblatts-Zitats registriert wird. Wie békannt, werden das $\mathbf{R}$ ichtersche Lexikon der Kohlenstoffverbindungen und die dazu erschienenen Supplemente den Mitgliedern der Deutschen Chemischen Gesellschaft durch die Verlagsbuchhandlung Leopold Voss in Hamburg zu einerh ermäßigten Preise geliefert.

Was den zweiten Teil des Antrages betrifft, so glaubt der Vorstand, dab gegenüber dem geringen Vorteil, welchen eine halbjährliche Registrierung der $\bowtie$ Berichto für den laufenden Jahrgang bringen würde, ein sehr viel größerer Nachteil für die dauernde Benutzung der Register entstehen würde, wenn der Inhalt eines Jahrgangs micht in ein em Register vereinigt, sondern auf $\mathrm{zw}$ wi Register vertoilt würde. Er sieht sich daher nicht veranlaßt, eine Änderung in dem gegenwärtigen Registriermodus dor Beriohtor vorzunehmen, und ist der Ansicht, dab den Wünacheren rascher Orientierung über die Erscheinuagen dos tafouden Jahres durob die den einzelnen Hoften der »Beriehte und des »Chemischen Zentralblatts《 beigegebenen Inhaltsverzeichnisse und durch die halbjährlichen Register des Zontralblatts genügend Rechnung getragen wird.

59. Die Sitzungen der Gesellschaft im Jahre 1908 sollen an folgenden Tagen stattfinden:

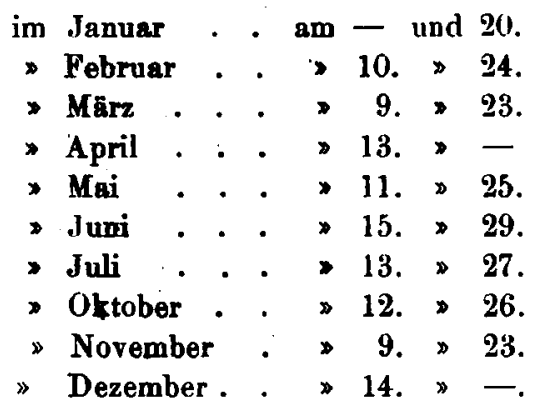

Der Vorsitzende:

Der Schriftführer:

S. Gabriel.

C. Schotter. 